\title{
Germanica
}

\section{Biologische Wahrnehmungsräume - zur Vegetation in der Sprache der Gegenwartslyrik}

Elisa Garrett : Espaces biologiques de perception - végétation et langage dans la poésie contemporaine

Elisa Garrett: Biological space of perception - vegetation and the language of contemporary poetry

\section{Elisa Garrett}

\section{(2) OpenEdition}

\section{Journals}

Édition électronique

URL : http://journals.openedition.org/germanica/6728

DOI : $10.4000 /$ germanica. 6728

ISSN : 2107-0784

\section{Éditeur}

Université de Lille

\section{Édition imprimée}

Date de publication : 26 juin 2019

Pagination : $91-100$

ISBN : 978-2-913857-42-1

ISSN : 0984-2632

\section{Référence électronique}

Elisa Garrett, „Biologische Wahrnehmungsräume - zur Vegetation in der Sprache der

Gegenwartslyrik", Germanica [Online], 64 | 2e trimestre 2019, Online erschienen am: 01 Januar 2021 abgerufen am 27 Februar 2021. URL: http://journals.openedition.org/germanica/6728 ; DOI: https:// doi.org/10.4000/germanica.6728 


\title{
Biologische Wahrnehmungsräume - zur Vegetation in der Sprache der Gegenwartslyrik
}

\author{
Elisa Garrett \\ Universität Bayreuth
}

Die Lyrik der heutigen Zeit präsentiert ein großes Spektrum an unterschiedlichen Gedichtformen. Besonders hervor sticht dabei die vegetative Struktur der Werke Nico Bleutges. Bleutges Gedichte liefern zunächst Inhalte, die in der Literatur spätestens seit dem 17. Jahrhundert vermehrt auftreten - Motive der Natur und eine wissenschaftsübergreifende Metaphorik aus dem Bereich der Botanik ${ }^{1}$. Einhergehend folgt die Erkenntnis, dass „Natur und Lebewesen nicht Konstrukte göttlicher Kunst sind, sondern dynamische, sich selbst gesetzmäßig organisierende Gebilde"2. Dieser Gedanke setzt sich im literarischen Schaffen fort. In Hinblick auf die Gestaltung des erzählten Raums tritt die Naturmetaphorik bei Bleutge in neuer Form und Funktion auf. Insbesondere lässt der im Jahr 2017 erschienene Gedichtband nachts leuchten die schiffe eine spezielle Beobachtungsform der Umgebung erkennen, die sich konkret als ,biologischer Blick‘ bezeichnen ließ $\mathrm{e}^{3}$.

1. — Vgl. Benjamin Bühler, „Botanik“, in: Roland Borgards u.a. (Hrsg.), Literatur und Wissen. Ein interdisziplinäres Handbuch, Stuttgart, Weimar, Metzler, 2013, S. 64-69, hier S. 66.

2. - Hubert Thüring, Das neue Leben. Studien zu Literatur und Biopolitik 1750-1938, München, Fink, 2012, S. 14.

3. - Vgl. Nico Bleutge, nachts leuchten die schiffe, München, C. H. Beck, 2017. 
Der vierte Gedichtband des Schriftstellers umfasst einen Zyklus aus zehn längeren Gedichten, die eine durchweg enge Verbindung zueinander aufweisen. Inhalt und Sprache folgen einem einheitlichen Duktus: Im Fokus steht die Vermittlung zwischen Mensch, Technik und Natur. Ausgangspunkt bildet der Bosporus in Istanbul als eine Sammelstelle für Ölcontainer und Handelsschiffe. Mittels detaillierter Beschreibungund Beobachtung wird reflektiert, wie Globalisierung und Welthandel Einfluss auf das wirtschaftliche Transportwesen ausüben und dabei die allgemeine Wahrnehmung von Zeit und Geschwindigkeit verändern. Handelsrouten sind gleichzeitig Fluchtlinien und tragen somit eine doppelte Bedeutung - hier vermischt sich die poetische Faszination des Meeres mit der Erinnerung an die eigene Kindheit und vergangene Tage. Bleutge selbst betont, dass in seinen Gedichten kein direktes Ich oder Wahrnehmungssubjekt vorhanden sei, sondern dass vielmehr eine „Wahrnehmungsmembran“ der Lyrik als Vermittler fungiert ${ }^{4}$. Folgt man diesem Begriff, ist die lyrische Sprache von einer Art autonomen Schutzhaut ummantelt; Sprache wird als biologischorganisches Phänomen modelliert. In den meisten Gedichtzyklen des Autors ist ein inhaltlich andauerndes "Eindringen der Vegetation“ $\mathrm{zu}$ vernehmen, was die einzelnen Gedichte als ein zusammenhängendes und biologisches Muster kennzeichnet ${ }^{5}$. Vegetation beschreibt zunächst die Gesamtheit der Pflanzenformation in einem bestimmten Gebiet - so ließe sich der Begriff in Übertragung auf den lyrischen Text als eine Sprachformation verstehen, die Nähe zum pflanzlichen Wachstum bietet. Thematisch liegt das Element des Vegetativen als sinnstiftendes Erzählmotiv vor und dient der Orientierung im Text. Hervorzuheben sind dabei die jeweils ersten Verse der einzelnen Gedichte. Diese formieren sich in Gesamtbetrachtung zu einem neuen und autonomen Erscheinungsbild: selbst das Inhaltsverzeichnis ist als nahezu eigenständiges Gedicht ganz in Bleutges Manier lesbar ${ }^{6}$. Die Vielfalt textueller Strukturen und ihrer Verknüpfungen, gepaart mit Begrifflichkeiten aus Biologie und Botanik, erscheinen als durchgehendes Erzählmuster des Gedichtbands. Die häufige Interdependenz von inhaltlicher und formaler Ebene verweist wiederholt auf die sprachliche Materialität des Werks und bildet eine auto-

Im Folgenden wird für den Verweis auf das Werk die Sigle $N B$ mit Seitenangaben verwendet.

4. - Tobias Lehmkuhl, „Das Wassergedicht. Über Steffen Popp, Nico Bleutge, Anja Utler“, in: Heinz Ludwig Arnold (Hrsg.), Junge Lyrik, München, edition text + kritik, 2006, S. 80-88, hier S. 85.

5. - Leonhard Hermann, Silke Horstkotte, Gegenwartsliteratur. Eine Einführung, Stuttgart, Metzler, 2016, S. 191.

6. - „versenk dich in die bewegung / öffne die tür / jetzt ist die nacht ein geräusch“ $(N B, 83)$. 
poietisch erweiterte Auffassung (lyrischer) Sprache - einer Sprache, die nicht nur in lyrischer Form vermittelt wird, sondern in den Gedichten selbst liegt.

\section{[denk wie / der tonsand, fern in der ahnung von muschelschichten]}

Diese Zeilen markieren eine besondere Verbindung zwischen Umgebungswahrnehmung und erzähltem Raum. Der Raum ist nicht durch eine beschreibende Instanz dargestellt, sondern fundiert auf einer Anweisung an die Wahrnehmung des Lesers: ,denk wie / der tonsand, fern in der ahnung von muschelschichten / denk wie muskeln und kalk“ $(N B, 8)$. Der anaphorische Aufruf ,denk wie“ führt die Ausgangsposition der Wahrnehmung direkt in die Struktur der Gedichte hinein. Dies geschieht durch die Nennung verschiedener Bodenrohstoffe, die in Bezug zum Organsystem der Muskulatur gesetzt werden. Tonsand und Kalk verorten die Textstelle zunächst im geologischen Wissensbereich und markieren die biogene Herkunft der Ressourcen. Die Muschel ist auf ihre zentralen Bestandteile von Muskel und Kalk reduziert und vermittelt eine Bewegung nach innen. Das sukzessive Eindringen ist durch das rhetorische Stilmittel der Paronomasie (Muschel/Muskel) verstärkt, indem zwei ähnlich klingende Begriffe knapp hintereinander aufgeführt sind und in ihrer zusammenhängenden Funktion bestärkt werden.

Der Übergang zwischen Außen- und Innenwelt setzt sich in den folgenden Versen fort: ,zelle um zelle / baute sich an, traum von geweben, häuten, wo du hinein- / gehst, siehst du nicht mehr hinaus, als wäre alles mit allem / verbunden, virus der weltpost" $(N B, 8)$. Mit der Betonung des ,traum[s] von geweben“ wird der Raum bildlich gerahmt und in den Bereich des Traumhaften verlagert. Ferner deutet die Verbindung von Muschel, Muskel, Gewebe und Häuten auf ein inneres Gleichgewicht des beschriebenen Organismus hin. Zwischen den Elementen besteht eine enge Verbindung, die auf die textuelle Struktur der Verse übertragbar ist. Die Verbindung der Wörter und Verse ist indexikalisch durch den Trennstrich (,hinein-gehst") betont und wird ebenso im Enjambement (,alles mit allem / verbunden“) markiert. Während der Trennstrich auf die Morphologie des Worts verweist, verbindet das Enjambement die Satzstruktur der Verse miteinander. Die Form der Gedichte spiegelt nicht nur die verstärkt wirtschaftlich orientierte Verbindung zwischen Mensch, Fortschritt und Natur wider, sondern dient auch der Veranschaulichung seiner eigenen Gegenständlichkeit: der sprachlichen Materialität der Lyrik. Auch die Metapher ,virus der weltpost“ lässt sich als Verweis auf die Sprache und den globalisierten Welthandel lesen. Zunächst erzeugt das Bild eine Assoziation mit einem Krankheitserreger, der sich global ausbreitet. Gleichzeitig markiert der Begriff „weltpost“ 
ein Symbol der grenzüberschreitenden Kommunikation durch Sprache und Schrift. Das kontinuierliche Wachstum des Handelsnetzwerks birgt Vorteile im Transportwesen, doch lässt es sich aufgrund seiner autonomen Verbreitung nicht aufhalten.

Es folgen weitere Anweisungen, die eine übergreifende Zustandsveränderung hervorrufen: ,,sei gischt / mit impulsen, sei staub, in nichts, ein büschel/wärme, versuche dorn zu sein und federn" $(N B, 15)$. Erneut liegt eine anaphorische Formulierung im Imperativ vor, die an den holistischen Effekt der vorherigen Verse anknüpft. Dabei wird mit unterschiedlichen Eigenschaften der Wahrnehmungsinstanz gespielt. Während die „gischt / mit impulsen“ eher eine dynamische Bewegung betont, lässt der inaktive ,staub“ einen Zustand des Stillstands erahnen. Ebenso stehen sich die Substanz der Gischt und die räumliche Leere (,in nichts") gegenüber. Die sprachlichen Gegensätze bestärken das ökonomische Zusammenspiel der Wahrnehmungsbereiche. Mit dem Begriff des „büschel[s]“ wird erstmals auf die Dominanz der pflanzlichen Natur verwiesen. Dies setzt sich in der Benennung von „dorn“ und „federn“ fort und mündet in dem Versuch, ein Empfinden in pflanzenund gräserähnlicher Form hervorzurufen. In Form des Oxymorons transportieren die Begriffe gleichzeitig Spitzes und Sanftes, wodurch der Ausdruck des Allumfassenden verstärkt wird. Während zu Beginn noch eher abstrakte Vergleichsmittel vorliegen, konkretisieren sich die Bilder im Verlauf zu greifbaren Inhalten. So manifestiert sich die Vorstellung biologischer (und chemischer) Einheiten beispielsweise in pflanzlichen ,färberdisteln“ und „fäden von chlorit“ $(N B, 15)$. Chemische und biologische Prozesse bestimmen den erzählten Raum durchgehend und konstruieren eine Form der Wahrnehmung, die eng mit der biologischen Materie verknüpft ist.

\section{[wenn du lange genug wartest / wachsen die schalen auf dem tisch weiter]}

Pflanzliche Schemata sind nicht nur begrifflich markiert, sondern dienen der Darstellung biologischer Prozesse. So heißt es in einer weiteren Beschreibung: ,wenn du lange genug wartest / wachsen die schalen auf dem tisch weiter / und die blätter in der hand werden zu gras / in dem du selber sitzt." $(N B, 11)$. Die Passage ist pflanzlich bestimmt und vermittelt Tendenzen der Vegetation. Der Raum umschließt das Zentrum „du“ von außen und innen, indem er gleichzeitig Ursprung und Hülle der Szene bildet. Statt sprunghaften Enjambements liegt ein parataktischer Aufbau der Verse im Zeilenstil vor. Dadurch werden die beschriebenen Zustände in einzelne Schritte unterteilt, die sich im Schriftbild spiegeln. Im Fokus steht die Synthese von Zentrum und Umgebung, die ein in sich verschränktes Raumsystem bildet. Eine 
ähnliche Formulierung lautet wie folgt: „mücken bilden mit ihren feinen kapseln die luft / die sie trägt.“ $(N B, 16)$. Die Umgebung (,luft“) geht aus dem in ihr Befindlichen („mücken“) hervor. Auch hier ist eine Synthese zwischen den inneren Elementen und dem umfassenden Raum erkennbar. Die Mücken erscheinen als „kapseln“ und tragen aktiv zur Bildung ihrer Umgebung bei. Die begriffliche Verbindung ließe sich als Angleichung tierischer und pflanzlicher Einheiten verstehen: in der Botanik dient die Kapsel der Kapselfrüchte als Samenhülle und ist die Grundlage für deren räumliche Ausbreitung7. Führt man diese Analogie fort, erscheint die Mücke ähnlich verschachtelt wie die Blätter und Gräser im Einklang von Ursprung und Umgebung. Luft und Raum sind vorwiegend mit pflanzlich-organischen Metaphern verbunden. Die Raumbildung erscheint als zyklische Bewegung, die dem Prozess biologischer Vegetation gleicht.

Des Weiteren zeigt sich die vegetative Gestaltung durch pflanzlich determinierte Konstruktionsmechanismen: „Mit blütensaft und braunen samen / mit spritzern von grün und wasserfäden / können die blätter des kautschukbaums, die sich selber zurück- / ziehen, wege wie luft in den raum zeichnen" $(N B, 13)$. Die vegetative Metaphorik bildet Verweise auf Blütenstand und Pflanzeninneres, die Betonung des ,samen[s]“ kann als Zeichen für beginnendes Leben gesehen werden. Diese Annahme wird durch den anaphorischen Ausdruck „mit" und der Benennung der „spritze[r] von grün“ verstärkt und verortet das Bild stark im Bereich vegetativer Farbmetaphorik. In Verbindung zur Farbe Grün stellt die ,spritzende" Bewegung inhaltliche Nähe zum Vitalismusgedanken her, nach dem die Lebenskraft als eigenständiges Prinzip gilt ${ }^{8}$. Diese Nähe zeigt sich ebenso im Element des Wassers. In den genannten Versen findet die Konkretisierung des Pflanzlichen im (aktiv agierenden) Kautschukbaum statt. Im Fokus steht abermals ein dynamischer Prozess, der von der sprachlichen Form unterstützt wird. Der Trennstrich dient einer Visualisierung des Begriffs: der Sprung in die nächste Zeile markiert eine Bewegung, die dem Vorgang des „zurück-ziehen[s]“ ähnelt. Erneut öffnet sich ein Zugang zur Morphologie der Wörter. Die Raumgestaltung ist durch bildnerische Verben wie „zeichnen“ unterstützt, die den Effekt der Konstruktion unterstützen. Ergänzend ist zu nennen, dass der letzte Teil des Verses ein weiteres Mal auftaucht. So heißt es an anderer Stelle: ,als würde das licht sich / verstärken, wege wie luft in den raum zeichnen“ $(N B, 8)$. Allein die Wiederholung identi-

7. - Vgl. Focko Weberling, Morphologie der Blüten und der Blütenstände, Stuttgart, Eugen Ulmer, 1981, S. 350.

8. - Vgl. Georg Toepfer, „Vitalismus“, in: Ders. (Hrsg.), Historisches Wörterbuch der Biologie. Geschichte und Theorie der biologischen Grundbegriffe, Bd. 3, Stuttgart, Metzler, 2011, S. 692-710. 
scher Verseinheiten ist als Verweis auf die sprachliche Materialität des Werks zu verstehen. In beiden Fällen handelt es sich um eine Integration der Raumbildung in die atmosphärische Wahrnehmung der Luft. Räumliche Strukturen sind weniger an fassbare Einheiten gebunden, sondern bilden sich im Zusammenspiel neu.

\section{[so wie ein tanker durch die helle wasserfläche gleitet]}

Die Ausbreitung und Konstruktion räumlicher Wahrnehmung geschieht vorwiegend durch organisch-vegetative Metaphern. Diese sind mit dem Element der Lebenskraft assoziiert und verbildlichen Prozesse biologischer Herkunft. Gleichzeitig verweisen sie auf die Ausbreitung des Wirtschaftssystems und die damit verbundene Erschließung neuer Handelsrouten über das Meer. Beobachtungen des Biologischen beziehen sich nicht nur auf die Pflanzenwelt, sondern sind insbesondere in einer Symbiose von Natur und Technik zu erkennen9. Beispielhaft stehen sich in folgender Passage Schiffskörper und biologische Beschreibung gegenüber: „so wie ein tanker durch die helle wasserfläche gleitet / zellhaut legt sich über zellhaut" $(N B, 7)$. Ähnlich der zentralen (Wahrnehmungs-)Membran übernimmt die Zellhaut eine schützende Funktion für die biologische Zelle, sie umschließt das Innere. Die Verse zeugen von einer Mehrlagigkeit, die einen kontinuierlichen Prozess des Schichtens in sich birgt. Tanker und Wasserfläche erscheinen als pflanzliche Einheit. Zudem betont der Vergleich zur Zellhaut die markante Stellung des Lebendigen: dem Vitalismusgedanken entsprechend dominiert das Lebendige gegenüber dem technischen Zustand und verweist somit auf die zunehmende Eigendynamik des internationalen Seehandels und des industriellen Transportwesens am Bosporus ${ }^{10}$.

Ähnlich zeigt sich die Verbindung von Technik und pflanzlichen Elementen in folgender Passage: ,die maschinen schlagen von unten an die schiffskörper / während das wasser schon seine wurzeln verliert" $(N B, 9)$. In diesem Ausschnitt funktioniert das Wasser als eigenständiger und vom Schiffskörper abgelöster pflanzlicher Organismus. Die Trennung ist durch den Versumbruch unterstrichen, wobei die lokaladverbiale Bestimmung ,von unten“ zu einer Positionierung unterhalb des Schiffs beiträgt. Personifizierte Maschinen „schlagen“ an den anthropomorphisierten ,schiffskörper“ und erscheinen als Störfaktor des Raumbilds. Auch die Begrifflichkeit der ,wurzeln“ kann als Metapher

9. — Vgl. Heide Kloth, „Konturen eines Ichs. Das Subjekt in der Lyrik Nico Bleutges“, in: Andrea Bartl (Hrsg.), Transiträume. Beiträge zur deutschsprachigen Gegenwartsliteratur, Augsburg, Wißner, 2009, hier: S. 314.

10. - Vgl. Tessy Korber, Technik in der Literatur der frühen Moderne, Wiesbaden, Springer, 1989, S. 129. 
des vegetativen Lebensbereichs verstanden werden. Das Verhältnis von Technik und Natur scheint kritisch - insbesondere das negativ konnotierte Verb ,verliert" trägt zum Eindruck einer Verlustsituation durch die dominante Wirtschaftsaktivität bei. Maßgeblich für die räumliche Erschließung und Ausbreitung des Seehandles ist ein biologisch geprägtes Bild der Bewegung:

[...] die erinnerungen drehen, dehnen sich langsam

als wären sie luftfäden, lebende moostierchen wie wanderbewegungen verloren gegangener handelsgüter, die das licht des tages aufsaugen und die frachtarbeiter an deck, ihre grellroten westen die noch kurz in der dämmerung wachsen $[\ldots](N B, 7)$

Zunächst entsteht der Wahrnehmungsraum auf Grundlage von Erinnerung. Die Faszination des Meeres als Ort internationalen Handels fließt mit der Erinnerung der beobachtenden Instanz zusammen. So vermittelt das Treiben am Bosporus ein Gefühl der Beständigkeit. Zeit und Geschwindigkeit lösen sich ineinander auf, während das Transportwesen einen dynamischen Prozess abbildet. Im wiederholten Stilmittel der Paronomasie (,drehen“ und „dehnen“) erscheint die Bewegung gleichzeitig linear und zyklisch. Die lineare Form wird durch die Betonung der „wanderbewegungen“" unterstrichen. Beide Begriffe vermitteln einen eher ruhigen und zwanglosen Eindruck. In den Versen liegt eine Art Gleichnis vor, das die „erinnerungen" durch ein konkretes Vergleichsbild darstellt. Der Vergleich beginnt zunächst in der Darstellung von „luftfäden“ und „lebende[n] moostierchen". Während die Luftfäden primär auf die atmosphärische Breite des Raums verweisen, öffnen die Moostierchen eine Nähe zum Organischen. Moostierchen sind bekanntlich vielzellige koloniebildende Tiere, die sich ähnlich der Pflanzenwelt durch ungeschlechtliche Fortpflanzung und Knospung vermehren. Sie erscheinen als tierischer und pflanzenähnlicher Organismus, dessen Lebendigkeit direkt benannt ist. Entsprechend ließe sich die Referenz auf die Moostierchen als vegetative Substantiv-Metapher verstehen, die das Bild der Erinnerung verstärkt und ein vegetatives Objekt der Bewegung markiert. Die Eigenschaften, die die Substantiv-Metapher konstituieren (,lebende“) sind ebenfalls vegetativer beziehungsweise vitalistischer Natur.

Ferner setzt sich der Vergleich in einer Übertragung auf „handelsgüter" und „frachtarbeiter" fort, wodurch ein Verweis auf Zivilisation und Ökonomie im Schiffsverkehr vorliegt. DieBeschreibung des Handels und der Menschen steht weiterhin in enger Verbindung zur Pflanzenwelt: Güter „saugen“ das „licht des tages“ auf und erinnern in metaphorischer Intensität des Ausdrucks an den Vorgang der Photosynthese und somit der Lichtaufnahme zur Nutzung der Lichtenergie. Ebenso stellen 
die „frachtarbeiter“ in „grellroten westen“ eine visuelle Verbindung zum Blütenstand her: der Zusatz des Wachsens in der ,dämmerung“ ist als Referenz auf das biologische Pflanzenwachstum lesbar. In ihrem Zusammenhang erzeugen die Verse einen vegetativen Raum, der durch dynamische Begrifflichkeiten unterstützt ist und auf den zyklischen Vorgang des biologischen Lebens verweist.

\section{lund wenn du an wörter wie schneeberg / denkst oder schlema]}

Während die organisch-vegetative Konstruktion des Raums zyklisch erfolgt, besteht auch der Gedichtband aus mehreren Zyklen. Neben der Interdependenz von sprachlicher und inhaltlicher Ebene taucht der Aspekt der sprachlichen Konzeption als Themenkomplex auf: „riecht wie heu, sagen die kinder, mit einem fluß dazwischen / und du weißt nicht, ob sie an wörter denken, an pflanzen / oder sich in die bewegung des wassers versenken“ $(N B, 11)$. Die Wahrnehmung beschränkt sich nicht nur auf das optische Erscheinungsbild, sondern wird durch die olfaktorische Modalität erweitert. Dabei herrscht eine enge Verbindung zwischen Wort, Pflanze und Wasser. Wort und Pflanze erscheinen auf einer Ebene und unterstreichen die autopoietische Form des Gedichts. Gleichzeitig transportieren die gewählten Begriffe eine Leichtigkeit der Bewegung, die sich in der rhythmischen Folge der Verse spiegelt. Darüber hinaus ist der wiederkehrende Effekt des inneren Einklangs zu erkennen: insbesondere das Präfix ver- im Verb „,versenken“ vermittelt eine Bewegungslinie nach innen. Zusätzlich liegt ein Verweis auf die sprachliche Materialität der Verse vor: der verborgene Reim zwischen „denken“ und „,versenken“ liefert Bezüge zur lyrischen Sprachnutzung.

Überdies heißt es im konkreten Bezug auf das Wort: „und wenn du an wörter wie schneeberg / denkst oder schlema, ziehst du langsam den rauch ein / und läßt die erinnerungen wachsen" $(N B, 13)$. Hier kommt dem Wort eine entscheidende Rolle zu. Mittels der Sprache entsteht eine räumliche Einheit, die in Verbindung zur zeitlichen Komponente und dem entsprechenden Vorstellungsbild der Erinnerung steht. Die Formulierung impliziert eine Aufforderung zur sprachlichen Reflexion. Der resultierende Denkprozess (,wenn du an wörter [...] / denkst") wird durch das Enjambement unterstrichen. Wirken die beiden „,wörter“ in ihrer Kleinschreibung zunächst unbedeutend und undefiniert, verweisen sie auf zwei relevante Orte im sächsischen Erzgebirge: Schon seit Jahrhunderten sind Schneeberg und Schlema für ihren florierenden Silberbergbau und ein besonders hohes Uranvorkommen bekannt. Die sprachliche Reflexion korrespondiert mit der Besinnung auf wirtschaftliche Entwicklung und deren Einfluss auf die gesellschaftliche Situation im globalisierten Zeitalter. Es wird 
ein Bewusstsein dafür geschaffen, wie sich die ökonomische Struktur zusammensetzt und sukzessiv zum alltäglichen Bestandteil des Lebens wird. Das Wachsen der Erinnerung folgt rückwirkend demselben Prinzip wie der Vorgang des Wirtschaftswachstums.

Ein direkter Bezug zur Erinnerung und ihrem Wachstumszyklus findet sich in den Versen ,die erinnerungen drehen, dehnen sich langsam / als wären sie luftfäden“ $(N B, 7)$. Zwischen den beiden Versgruppen zeigen sich deutliche Parallelen. Generell weist die Bewegung des Zyklischen nicht nur auf den Fortlauf des Lebens hin, sie bedingt ebenso den Effekt des räumlichen und begrifflichen Einklangs: „wo die strahlen das eismeer erkunden / und die fische sich in fische auflösen, eine bewegung / die keinem traum folgt, erst sichtbar wird im verschwinden" $(N B, 9)$. Neben dem Paradoxon der Fische findet ein Übergang in den Bereich der Imagination statt. Sie ist durch die doppelte Negation („,keinem traum“) und der paradoxen Definition des Sichtbaren (,im verschwinden") unterstützt. Die beschriebene Bewegung bleibt unkenntlich und zeigt sich ausschließlich in den Worten selbst. Gleichzeitig wird der Effekt des Einklangs visualisiert, der einem Vorgang des ,zurück in sich selbst“ folgt $(N B, 10)$. Raum und Biologie markieren einen dynamischen Prozess, der sich wiederholt in der Struktur der Worte und Verse spiegelt. Die Idee des Einklangs steht demnach im konkreten Bezug zur Vegetation:
wo sich die pflanzen mischen, windstille, erdstoff mühle, wo die pigmente trocknen und das wasser langsam verkocht. als schlehe, mit einem kurzen blühen, kann sich das gesicht des schlafenden weiß färben. was er träumt, im erkunden erdwärts wird von den blättern aufgesaugt $[\ldots](N B, 15)$

Die Verse vermitteln eine Übertragung der pflanzlichen Vegetation auf den umgebenden Raum. Dieser impliziert nicht nur verschiedene Formen der Wahrnehmung, sondern betont gleichzeitig die Präsenz einer Wahrnehmungsinstanz. Der beschriebene Vorgang des „mischen[s]“ ist durch die zahlreichen Enjambements gekennzeichnet und visuell im Sprachbild markiert. Im fließenden Übergang der Verse werden die Strukturen der abstrakten Sätze sichtbar. Auffällig ist, dass in den meisten Teilen des Gedichtbands keine Satzzeichen verwendet werden. Hier jedoch liegen zwei Endpunkte innerhalb der Verse vor und suggerieren eine sprachliche Einheit. Die Satzzeichen verweisen nicht nur auf die sprachliche Materialität des Gedichts, sondern tragen in nun hypotaktischer Versstruktur zum Effekt der strukturellen Vermischung bei. Diese Bewegung ließe sich ähnlich der Vegetation und der kontinuierlichen Vernetzung des Wirtschaftssystems verstehen. Konkrete Bezüge bietet die Figur des ,schlafenden“- das reell benannte „gesicht“ wird 
zuvor durch die vegetative Metapher als weiße Blüte des Schlehdorns visualisiert. Ebenso ist ein beständiges Bild der inneren Bewegung und des harmonischen Einklangs der Verse gegeben. Der Gedichtzyklus markiert eine Verwandtschaft zwischen Mensch und Pflanze. Diese Verbundenheit korrespondiert mit der lyrischen Sprache und löst die Grenzen von ,Ich ' und ,Welt' zunehmend auf. Dadurch entsteht der Effekt einer in sich geschlossenen Wahrnehmung, die der Vorstellung einer lyrischen Wahrnehmungsmembran nahekommt.

Impressionen des Vegetativen ziehen sich durch den gesamten Zyklus hindurch und bestimmen sowohl Inhalt als auch sprachliche Form der Gedichte. Die zahlreichen Metaphern bedingen eine schöpferische Umwandlung der Worteinheiten, die vorwiegend biologischer Herkunft sind und dem naturwissenschaftlichen Wissensbereich entstammen. Die Effekte des Vegetativen dienen nicht nur als gestalterisches Mittel des Wahrnehmungsraums, sondern markieren die sprachliche Materialität der Verse. Gleichzeitig zielen sie auf die übergeordnete Bedeutung des wachsenden Handelsnetzwerks auf See und dessen Einfluss auf die zeitliche und räumliche Wahrnehmung ab. Begriffsbeziehungen und Wortverbindungen erscheinen als biologisch-organische Muster und verweisen auf die vegetative Struktur de Lyrik. Während die Pflanze als Symbol der Sprache hervortritt, funktioniert der pflanzliche Aufbau als Entität der Sprachbildung. Bleutge lässt nicht nur einen Raum entstehen, der auf biologische Weise erfasst werden kann: der Gedichtzyklus nachts leuchten die schiffe kennzeichnet vielmehr einen intensiv vegetativen Sprachstil, der als Allegorie an den Prozess des Dichtens und die wirtschaftliche Entwicklung des globalisierten Zeitalters anklingt. 